\title{
Revealing Disturbance Localization Issues in Large Power Systems
}

\author{
Urban Rudez $^{1}$, Rafael Mihalic ${ }^{1}$
}

\begin{abstract}
Most of European Transmission System Operators report positive experience concerning national Wide Area Monitoring System (WAMS). Currently, WAMS is mainly used for off-line analysis of past events; however, few reports of its use in the process of real-time decisionmaking can also be found in the literature. As TSOs confidence in this technology increases, several important steps have already been made towards implementing WAMS as a permanent tool for operator's real-time assistance. In this paper, issues concerning electromechanical disturbance localization in large electric power systems are enlightened and explained. Research so far showed that the task is far from being trivial. Common localization techniques such as multilateration might exhibit unacceptable errors in determining geographical location of the source. Namely, disturbance propagation speed can vary up to several times, depending on the electrical network specifics. This is why the quest for alternative approaches to localization is underway in order to increase its accuracy to a satisfactory level.
\end{abstract}

\section{Keywords}

WAMS, electromechanical disturbance propagation speed, event localization.

\section{Acknowledgement}

Authors would like to thank company ELPROS for their

\footnotetext{
${ }^{1}$ Department of Power Systems and Devices

Faculty of Electrical Engineering

University of Ljubljana

1000 Ljubljana, Slovenia

(e-mail: urban.rudez@fe.uni-lj.si, rafael.mihalic@fe.uni-lj.si)
}

assistance by enabling access to WAProtector application.

\section{Introduction}

Wide Area Monitoring System (WAMS) technology can already be considered as a well-established technology among several Transmission System Operators (TSOs) around the world. Especially in Europe, TSOs show high level of confidence that WAMS can efficiently be used for real-time protection and control applications (Wide Area Monitoring, Protection and Control - WAMPAC). Slovenian TSO ELES is among most advanced European TSOs in this area with an extremely high share of high-voltage bus Phasor Measurement Unit (PMU) coverage. WAMS concept is said to enable the following [1]:

- real-time system state estimation,

- real-time congestion management

- real-time phase angle monitoring,

- real-time disturbance propagation monitoring,

- protection for wide-area disturbances (system protection schemes),

- estimation of the load model parameters,

- validation of generator models,

- system operation closer to stability limits,

- real-time control of FACTS devices based on global system conditions.

This paper deals with the fourth bullet, which is inevitably associated with localization of disturbances, such as large generation unit outages, outages of fullyloaded transmission lines, etc. Such events cause electromechanical system responses (synchronous generator swings). Several publications can be found in the literature dealing with the subject of

This is an Open Access article distributed in accordance with the Creative Commons Attribution Non Commercial (CC-BY-NC-ND 4.0) license, which permits others to copy or share the article, provided original work is properly cited and that this is not done for commercial purposes. Users may not remix, transform, or build upon the material and may not distribute the modified material (http://creativecommons.org/licenses/by-nc/4.0/) 
electromechanical disturbance origin localization, a common point of all being that this can hardly be considered a simple task due to:

- electromechanical disturbance propagation speed is not constant ([2], [3], [4]). Instead, it depends on system impedances and generation units' inertia. This is the reason why simple localization techniques such as multilateration [5] are not suitable for the task. Fig. 1 shows an example of different propagation speeds in the opposite direction between two locations, observed in the USA [4],

- no direct relation was found so far between characteristics of oscillating system variables and the moment of disturbance occurrence. This is why WAMS applications that enable real-time oscillation monitoring are not yet capable of localizing a root event causing the oscillation.

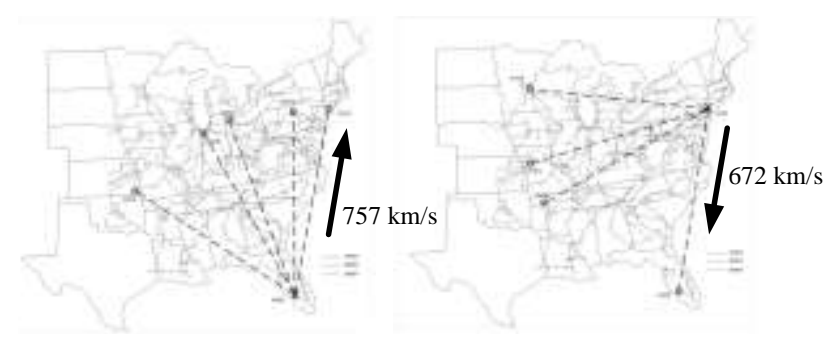

Fig. 1 Electromechanical disturbance propagation speed depends on propagation direction, experience from USA [4]

At first, physical background of electromechanical disturbance propagation should be briefly described in order to be able to identify most important influential system parameters.

\section{Influential parameters}

An interesting approach to power-system modelling for analysing this issue is a continuous distribution of all power-system elements ([6]-[11]). This is very common for transmission line modelling (observation of infinitely small line fraction $\mathrm{d} x$ ), whereas it is quite unrealistic for synchronous generator parameters (including inertia); a space-discrete distribution would a realistic choice. Despite all, such modelling gives one a splendid insight into the phenomenon of electromechanical disturbance propagation. Namely, this phenomenon can be described as a wave, with the following propagation speed:

$$
v=\sqrt{\frac{\omega V^{2} \sin \Theta}{2 h \cdot|z|}}
$$

where $\omega$ is a nominal system frequency in radians, $\Theta$ impedance angle in radians, $V$ voltage amplitude in p.u., $h$ inertia constant in seconds per unit length $\mathrm{d} x$ and $|z|$ total line impedance in seconds per unit length $\mathrm{d} x$. As system voltages can be considered close to 1 p.u., most influential parameters appear to be line impedance $z$ and generator inertia $h$. As long as homogenous system is considered, $v$ appears to be the same all over the system. Multilateration technique can therefore be efficiently used. On the other hand, in reality both $z$ and $h$ vary from one part of the system to another, which is the reason for an emerging need for alternative localization approaches. These approaches vary from mathematically exact yet complex [12] which makes them less suitable for practical implementation up to more practically oriented [13]. However, both require profound understanding of the phenomenon [14].

In order to approach more realistic circumstances, it is reasonable to apply a discrete radial system from Fig. 2. A disturbance is simulated on the far left-hand side end of the model as a sudden step change in phase angle $\delta_{0}$.

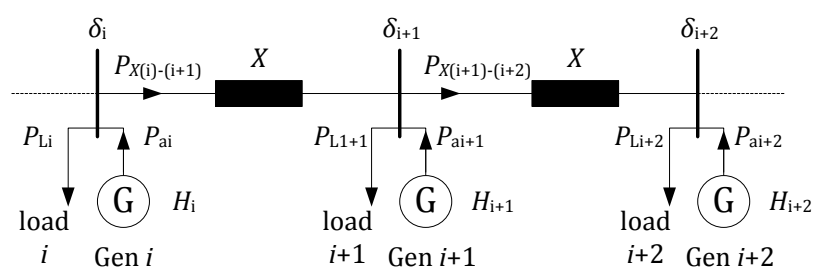

Fig. 2 Applied radial test system

First, it is reasonable to provide two most important equations required for understanding the phenomenon. Each change in phase angle (e.g. $\delta_{\mathrm{i}}$ ) causes the active power flow (e.g. $P_{\mathrm{X}(\mathrm{i}) \text {-(i+1) }}$ ) to change according to:

$$
P_{X(i)-(i+1)}=\frac{U_{\mathrm{i}} \cdot U_{\mathrm{i}+1}}{X} \sin \left(\delta_{\mathrm{i}}-\delta_{\mathrm{i}+1}\right)
$$

As soon as the power flow changes, power balance in the neighbouring bus (e.g. bus $i+1$ ) and consequently on the corresponding generator terminals (e.g. Gen $i+1)$ is disturbed. This means that the accelerating power (e.g. $\left.P_{\mathrm{a}, \mathrm{i}+1}\right)$ appears on the synchronous generator which reacts according to swing equation:

$$
\frac{2 H_{\mathrm{i}+1} \cdot S_{\mathrm{n}, \mathrm{i}+1}}{\omega_{\mathrm{n}}} \cdot \ddot{\delta}_{\mathrm{r}, \mathrm{i}+1}=P_{\mathrm{a}, \mathrm{i}+1}
$$

As each phase angle (e.g. $\delta_{\mathrm{i}+1}$ ) is strongly related to corresponding generator rotor angle (e.g. $\left.\delta_{\mathrm{r}, \mathrm{i}+1}\right)$, the phase angle eventually also changes which transfers the same effect towards the next generator in chain.

In order to show how $X$ and $H$ influence this electromechanical disturbance propagation, two analyses 


\section{U. Rudez, R. Mihalic \\ Revealing Disturbance Localization Issues in Large Power Systems}

were performed. First, inertia constant of Gen $10\left(H_{10}\right)$ and second, reactance of line between busses 9 and 10 $\left(X_{9-10}\right)$ was varied. In the first case, $H_{10}$ was set to higher (Fig. 3) / lower (Fig. 4) value compared to other generators and similar was done for reactance $X_{9-10}$ (see Fig. 5 and Fig. 6). With the solid curves results with equal parameters across the entire system are presented, whereas with dashed curves with a corresponding parameter (either $H_{10}$ or $X_{9-10}$ ) being varied. In general, results indicate that higher values of inertia appear as if the wave is being delayed and lower in amplitude. On the other hand, lower values of inertia indicate quite the opposite. The same conclusion can be drawn for variations of line reactance.

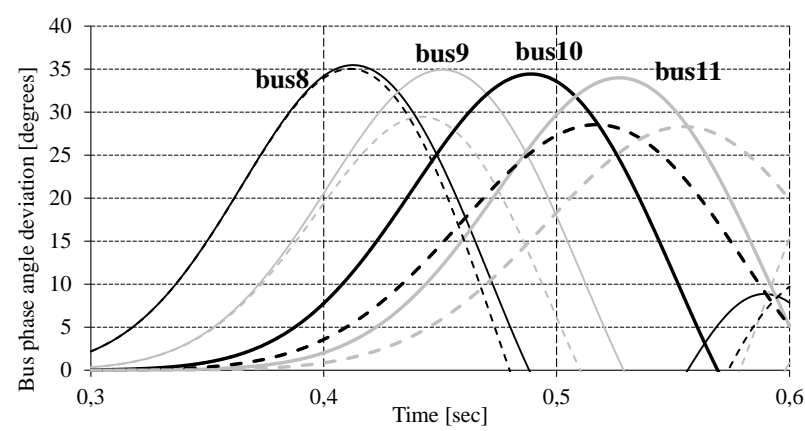

Fig. 3 Impact of inertia constant variation on electromechanical disturbance propagation (high $H_{10}$ )

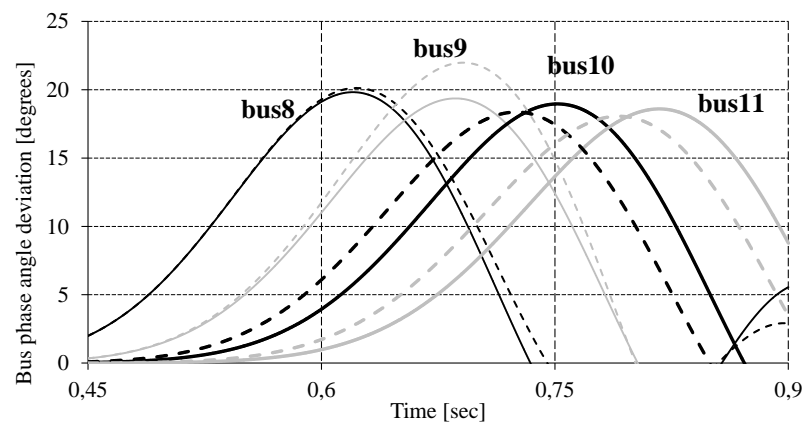

Fig. 4 Impact of inertia constant variation on electromechanical disturbance propagation (low $H_{10}$ )

Besides mentioned two system parameters, the vicinity of network limits (borders) also influences the wave amplitude and consequently also the detection of its arrival at the certain location. It is often forgotten that despite interconnections might span over entire continents, they still have their physical borders. Similar than in case of a mechanical wave propagation on a simple rope (physical analogy), in power systems the amplitude also significantly increases in the vicinity of the border - see Fig. 7. On the horizontal axis, a sequence of radial network busses are provided, whereas on the vertical axis a phase-angle deviation is depicted from their pre-fault values. With the grey curve phase-angle deviations are depicted 1.2 seconds after fault occurrence. It is clear that phase angles oscillate with certain time delays which makes the situation appear as a travelling wave (so-called electromechanical wave). A solid black curve represents the amplitude of the first wave in series for homogeneous conditions all over the system. It can be observed that at the 64-bus radial network border the wave amplitude increases to approximately twice the value. Dashed and dotted black lines show the impact of increasing/decreasing inertia constants of generators from 50 to 64 and reactances of lines between busses 50 to 64 .

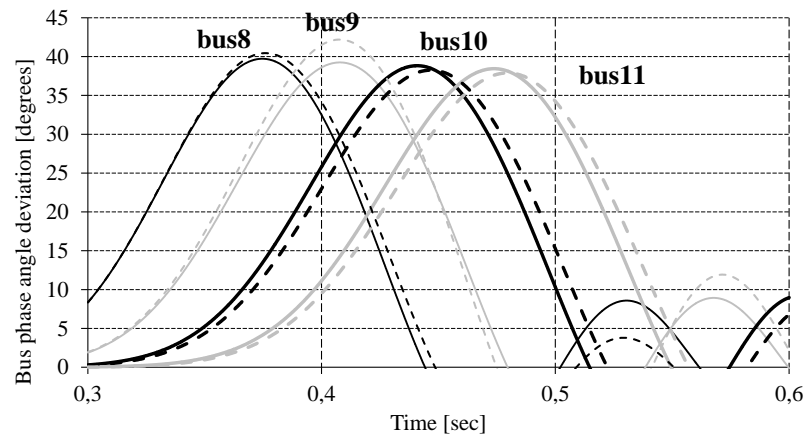

Fig. 5 Impact of line reactance variation on electromechanical disturbance propagation (high $X_{9-10}$ )

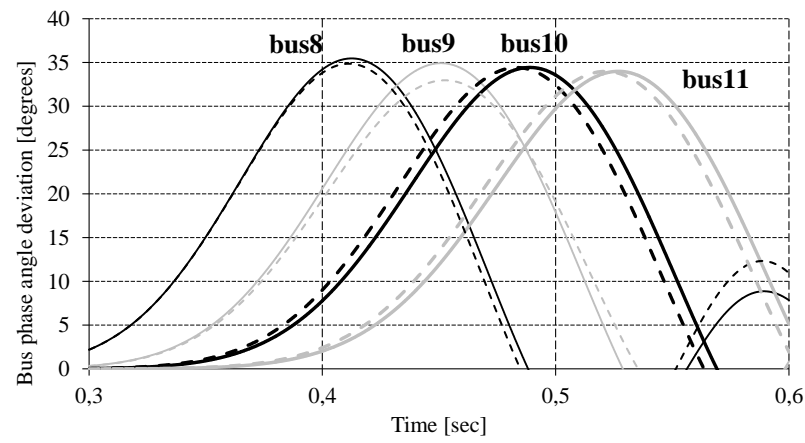

Fig. 6 Impact of line reactance variation on electromechanica disturbance propagation (low $X_{9-10}$ )

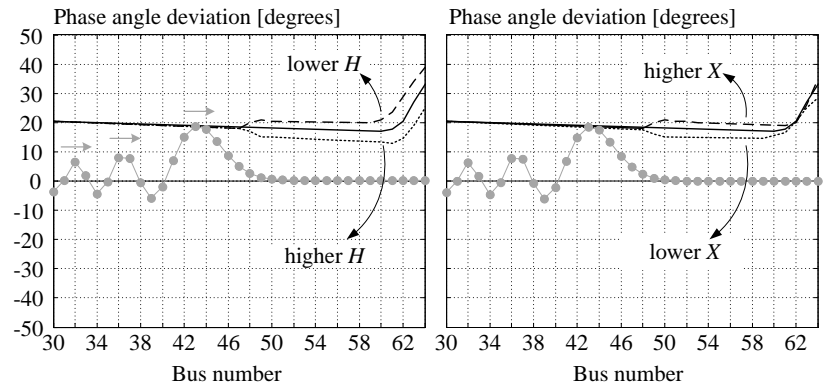

Fig. 7 Impact of inertia constants $H$ and line reactances $X$ on electromechanical wave amplitude at the vicinity of network borders (left: impact of $H$, right: impact of $X$ ) 


\section{Measuring Time of Arrival}

At the first sight, measuring the Time of Arrival (TOA) of an electromechanical wave appears as a very trivial problem. Yet it was found to present a very serious challenge. As long as one operates with discrete signals, determination of TOA is straightforward. On the other hand in case of continuous-changing signals (e.g. Fig. 3 to Fig. 6) this is no longer the case. Generally, there are three possibilities to approach this issue:

- setting the fixed threshold value (TOA is set when the deviation of observed variable exceeds a pre-defined threshold value. This is most commonly used approach, also in FNET application in the USA),

- observing wave peak value (TOA is set when the deviation of observed variable reaches its peak value),

- observing wave bifurcation point (TOA is set when the second derivative of deviation of observed variable reaches its peak, i.e. peak value of its acceleration).

In systems, where the wave propagation speed all across the system maintains at a constant speed, it is possible to successfully implement a multilateration concept for wave origin localization. For this purpose, the term Time Difference Of Arrival (TDOA) is often used in the literature. However, Fig. 3 to Fig. 6 showed that electromechanical wave shape is strongly influenced by the system parameters, which vary from one part of the system to another. So it is clear that TOA measuring with a fixed threshold might encounter severe issues if the threshold is set too high (which is what is actually expected in practice due to the constant presence of noise and very low-frequency oscillations in PMU phase-angle measurements). Of course this issue is irrelevant when dealing with noiseless simulations, but enables one to observe how TOA is affected when $H$ and $X$ are varied in the radial system - see Fig. 8. With the dashed grey curve TOA results with equal parameters in the entire system are presented. TOA appears to linearly increase with the increasing distance from the disturbance origin. On the other hand, higher $H_{8}$ up to $H_{13}$ cause the TOA between two subsequent busses to be higher (apparent deceleration of the wave). Similarly, lower $X_{18-19}$ to $X_{22-23}$ cause the TOA between two subsequent busses to be lower (apparent acceleration of the wave). So it is obvious that localization techniques that operate without considering network parameters in general cannot give competent results. At this point a question arises as to what is the error they produce during localization and whether it is perhaps negligible?

\section{Practical examples: multilateration}

In this section, multilateration-based localization results for two actual events in ENTSO-E interconnection are presented. As already written, multilateration is a typical representative of a technique that does not consider electrical system parameters. Instead, it operates only on knowing geographical distances between measuring points and considering wave DTOA between them. For the two presentedcases, input PMU data was provided by the WAProtector application, produced in the company ELPROS, Slovenia [15]. Measurements from six locations in ENTSO-E were available, marked with round dots in Fig. 9.
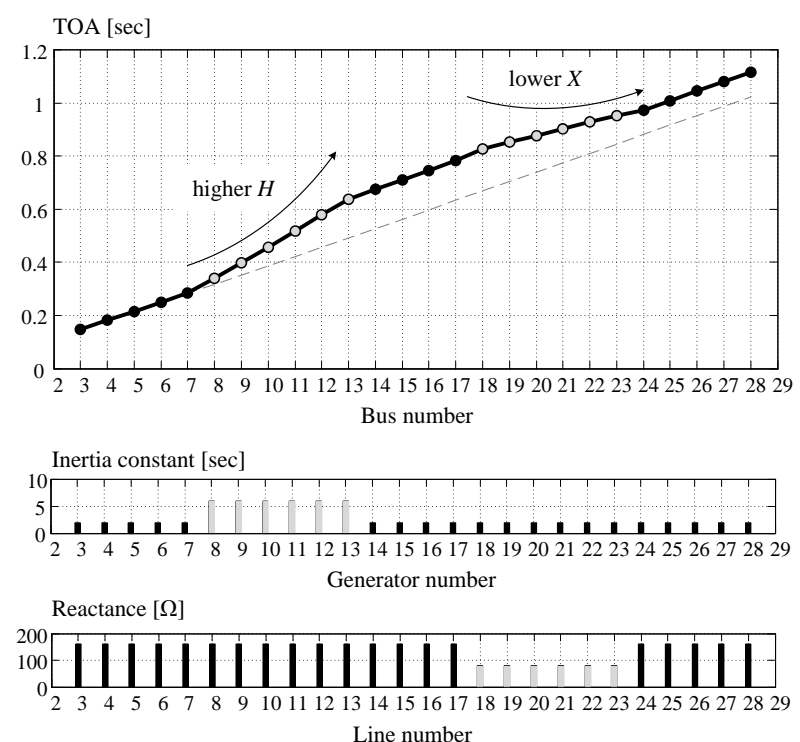

Fig. 8 Example of inertia constant and line reactance impact on electromechanical disturbance propagation speed measurement

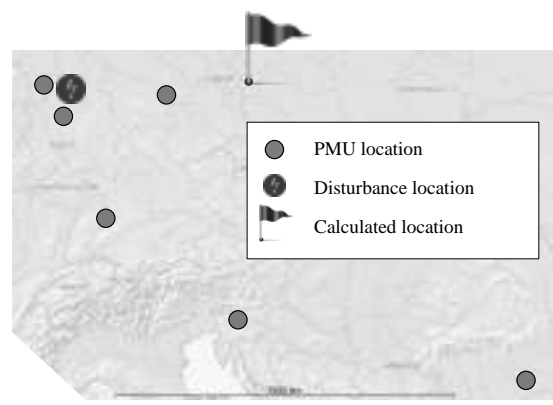

Fig. 9 Localization results: Ibbenburen generation unit outage, Germany, $21^{\text {st }}$ March 2015

Due to the presence of noise and the finite input data resolution of $20 \mathrm{~ms}$, few signal manipulations were first required. Usually, simple procedures such as higher-order polynomial approximation within selected data window are effective in such cases. However, the selection of appropriate approach in this phase is very important, as 
the multilateration procedure is very sensitive to discrepancies in DTOA. Namely, DTOA is the sole data set required by this technique. This is clearly evident from the generation unit Ibbenburen (Germany) outage that took place on 21st March 2015 (Fig. 9). $7^{\text {th }}$ order polynomial approximation obviously brought a quite significant error in the procedure. The localization procedure was disturbed to such an extent that it pointed to a location approximately $500 \mathrm{~km}$ eastern from the actual event taking place.

Next, a geographical location of PMU devices also plays an important role in multilateration concept. This can be confirmed by analysing the case of generating unit Wilhelmshaven (Germany) outage that took place on 22nd March 2015 (Fig. 10). It turns out that a disturbance in both actual and calculated location produces similar DTOA values. As this is the sole input to the procedure it is reasonable to expect significant errors, as only the direction towards the source can be determined and not its distance. This was confirmed in Fig. 10 where the calculated location was not only $2000 \mathrm{~km}$ north-eastern from the actual location, but also in the other synchronous system.

Authors believe that two presented examples clearly show the need for alternative localization technique that is not based merely on geographical locations of PMU devices but also takes system parameters into account.

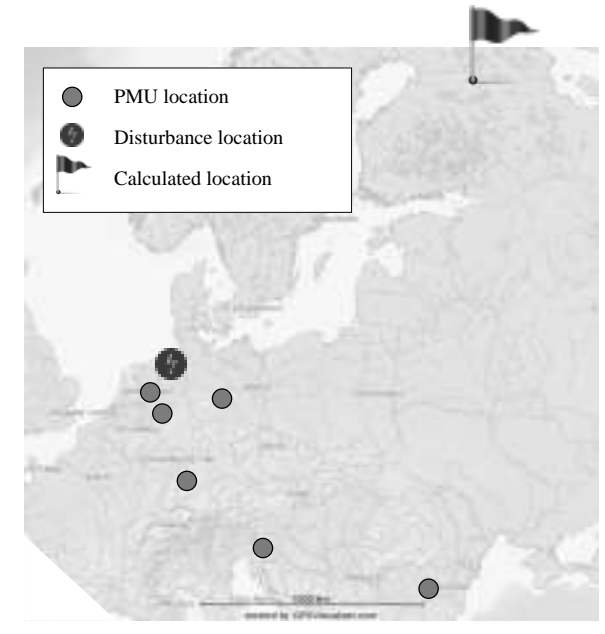

Fig. 10 Localization results: Wilhelmshaven generation unit outage, Germany, 22 ${ }^{\text {nd }}$ March 2015 (right)

\section{Conclusion}

With the final overall acceptance of WAMS technology, the next logical step appears to be using WAMS for protection and control purposes in real-time. One of most important features of such WAMPAC application would be the detection and localization of large events, which are more or less constantly taking place in an interconnected power system. Information about the origin of the disturbance would represent a significant factor in the process of determining possible system interventions to retain system integrity.

In this paper, most important influential factors are presented that play a vital role in electromechanical disturbance propagation across the system. A specific space-distributed mathematical modelling of the entire system (including generators' inertia) enable analytical derivation of the expression for the electromechanical wave propagation speed. It turns out that generator inertia constants and transmission lines reactance play most important role. This is why a special analysis was conducted on a 64-generator radial power-system dynamic model in order to further investigate influence of these two parameters. According to the results it can be concluded that the fact of these two parameters not being constant all across the system should not be carelessly neglected. This is why authors express their concern regarding the use of localization procedures such as multilateration that does not take into account any information about the system. Instead, they are based merely on geographical distance between PMU units and measured DTOAs. Authors' reservations are supported by two examples of the actual events taking place in ENTSO-E interconnection in March 2015. This is why a research is under way for finding alternative localization techniques that would consider system parameters as well. In this way, localization would be strongly improved and eventually reached such level of efficiency that would be acceptable by the TSOs.

\section{References}

[1] V. Terzija, G. Valverde, D. Cai, P. Regulski, V. Madani, J. Fitch, S. Skok, M. M. Begovic, and A. Phadke, "Wide-Area Monitoring, Protection, and Control of Future Electric Power Networks," Proceedings of the IEEE, vol. 99, no. 1, pp. 80-93, Jan. 2011.

[2] A. J. Arana, J. N. Bank, R. M. Gardner, and Y. Liu, "Estimating Speed of Frequency Disturbance Propagation Through Transmission and Distribution Systems," in Power Systems Conference and Exposition, 2006. PSCE '06. 2006 IEEE PES, 2006, pp. 1286-1290.

[3] K. S. Kook, Y. Liu, and M. J. Bang, "Global behaviour of power system frequency in korean power system for the application of frequency monitoring network," IET Generation, Transmission Distribution, vol. 2, no. 5, pp. 764-774, Sep. 2008.

[4] S. S. Tsai, L. Zhang, A. G. Phadke, Y. Liu, M. R. Ingram, S. C. Bell, I. S. Grant, D. T. Bradshaw, D. 
Lubkeman, and L. Tang, "Study of global frequency dynamic behavior of large power systems," in Power Systems Conference and Exposition, 2004. IEEE PES, 2004, pp. 328-335 vol.1.

[5] "Multilateration," Wikipedia, the free encyclopedia. 01-Apr-2014.

[6] A. Semlyen, "Analysis of Disturbance Propagation in Power Systems Based on a Homogeneous Dynamic Model," IEEE Transactions on Power Apparatus and Systems, vol. PAS-93, no. 2, pp. 676-684, Mar. 1974.

[7] J. S. Thorp, C. E. Seyler, and A. G. Phadke, "Electromechanical wave propagation in large electric power systems," IEEE Transactions on Circuits and Systems I: Fundamental Theory and Applications, vol. 45, no. 6, pp. 614-622, Jun. 1998.

[8] D. Wang and X. Wang, "Analytical study on electromechanical wave propagation in a nonuniform continuum power system," in Third International Conference on Electric Utility Deregulation and Restructuring and Power Technologies, 2008. DRPT 2008, 2008, pp. 958-963.

[9] M. Parashar, J. S. Thorp, and C. E. Seyler, "Continuum modeling of electromechanical dynamics in large-scale power systems," IEEE Transactions on Circuits and Systems I: Regular Papers, vol. 51, no. 9, pp. 1848-1858, Sep. 2004.

[10] A. J. Thomas and S. M. Mahajan,
"Electromechanical Wave Analysis Through Transient Magnetic Modeling," IEEE Transactions on Power Delivery, vol. 24, no. 4, pp. 2336-2343, Oct. 2009.

[11] D. Wang, X. Wang, and J. S. Thorp, "Study on electromechanical wave continuum model for power systems in mechanics," in IEEE Power Engineering Society General Meeting, 2006, 2006, p. 9 pp.-.

[12] S. Backhaus and Y. Liu, "Electromechanical Wave Green's Function Estimation from Ambient Electrical Grid Frequency Noise," arXiv:1108.1804 [physics], Aug. 2011.

[13] U. Rudez and R. Mihalic, "A method of detecting the time of arrival for an electromechanical wave in large power systems," in PowerTech (POWERTECH), 2013 IEEE Grenoble, 2013, pp. 16.

[14] U. Rudez and R. Mihalic, "Understanding the electromechanical wave propagation speed," in Bulk Power System Dynamics and Control - IX Optimization, Security and Control of the Emerging Power Grid (IREP), 2013 IREP Symposium, 2013, pp. 1-8.

[15] Tadeja Babnik and Bojan Mahkovec, "WAProtector," presented at the PIES 2010, Nov2010. 